\title{
Output Maximal Dimension for the Disturbance Decoupling Problem
}

\author{
F.Puerta $^{(1)}$ - X.Puerta ${ }^{(2)}$ - I.Zaballa ${ }^{(3)}$ \\ (1) ETSEIB, Departament de Matemàtica Aplicada I. Universitat Politècnica de Catalunya \\ Diagonal 647 -08028 Barcelona - Spain. e-mail: puerta@ma1.upc.es \\ (2) EUPB, Departament de Matemàtica Aplicada I. Universitat Politècnica de Catalunya \\ Av. Gregorio Marañón -08028 Barcelona - Spain. e-mail: coll@ma1.upc.es \\ (3) Departamento de Matemática Aplicada y EIO. Universidad del País Vasco \\ Apartado 640, 48080 Bilbao - Spain. e-mail: izaballa@picasso.lc.ehu.es
}

\begin{abstract}
Given a linear time invariant system with a disturbance we describe a method of finding the maximal dimensional output for a generic Disturbance Decoupling Problem.
\end{abstract}

Keywords: Linear system. Brunovsky canonical form. Thom transversality theorem. Disturbance decoupling problem.

\section{Introduction}

Given the control linear system

$$
\begin{aligned}
\dot{x}(t) & =A x(t)+B u(t)+Q q(t) \\
y(t) & =C x(t)
\end{aligned}
$$

the term $q(t)$ represents a disturbance which is assumed not to be directly measurable by the controller. Then, the Disturbance Decoupling Problem (DDP) consists in finding (if possible) a state feedback $F$, such that the disturbance $q(t)$ has no influence on the output. That is to say, $F$ must be such that for any initial state the corresponding output $y(t)$ of the system

$$
\begin{aligned}
& \dot{x}(t)=(A+B F) x(t)+B u(t)+Q q(t) \\
& y(t)=C x(t)
\end{aligned}
$$

does not depends on $q(t)$.

A solution of this problem can be found for example in [5], Th.4.2, where the following result is proved: the DDP has a solution if and only if

$$
\mathfrak{Y}(A, B ; \operatorname{Ker} C) \supset \operatorname{Im} Q
$$


where $\mathfrak{Y}(A, B$; Ker $C)$ is the unique maximal $(A, B)$-invariant subspace contained in Ker $C$. We recall that a subspace $S$ is said to be $(A, B)$-invariant if $A(S) \subset S+\operatorname{Im} B$.

In this note we tackle the problem of showing a method of finding, for a generic $Q$ the maximal dimension $p$ of the output space insensitive to the disturbance $q(t)$. We will call briefly this problem "A maximal solution of the DDP". In [4] is showed through an example a method of solving this problem when the pair $(A, B)$ is controllable. In this note we solve this problem when $(A, B)$ is a general pair. For this we make use of the bundle structure of the set of conditioned invariant subspaces given in [2].

We fix the following notation. $\mathcal{M}_{p, q}$ will denote the set of complex matrices having $p$ rows and $q$ columns. We write $\mathcal{M}_{p}$ for $\mathcal{M}_{p, p}$. If $A \in \mathcal{M}_{p}$ we say that $A$ is a $p$-matrix. $\mathcal{M}_{p, q}^{*}$ is the set of full rank matrices in $\mathcal{M}_{p, q}$.

If $A \in \mathcal{M}_{p, q}, A^{*}$ means the conjugate transpose of $A . I_{n}$ will be the identity matrix. If $A \in \mathcal{M}_{p, q},[A]$ means the subspace generated by the columns of $A$.

If $E$ is a vector space, $\operatorname{Gr}_{d}(E)$ is the Grassman manifold of $d$-dimensional subspaces of $E$.

A Brunovsky (dual) matrix, $B$-matrix in what follows, is a matrix of the form $\left(\begin{array}{l}N \\ E\end{array}\right)$ where $N=\operatorname{diag}\left\{N_{0}, N_{\infty}\right\}, E=\operatorname{diag}\left\{E_{0}, 0\right\}$, being $N_{\infty}$ a Jordan matrix and $\left(E_{0}, N_{0}\right)$ a Brunovsky observable pair, that is to say, $N_{0}=\operatorname{diag}\left\{N_{1}, \ldots, N_{r}\right\}$ each $N_{i}$ being the standard lower nilpotent $k_{i}$-matrix, $E_{0}=\operatorname{diag}\left\{E_{1}, \ldots, E_{r}\right\}$, each $E_{i}$ being a $k_{i}$-row matrix, $E_{i}=(0, \ldots, 0,1), 1 \leq i \leq r$. The integers $k_{1}, \ldots, k_{r}$ are called the observability indices of $\left(\begin{array}{l}N \\ E\end{array}\right)$.

\section{A maximal solution of the DDP}

(2.1) Consider a linear system

$$
\begin{aligned}
\dot{x}(t) & =A x(t)+B u(t)+Q q(t) \\
y(t) & =C x(t)
\end{aligned}
$$

where $A \in \mathcal{M}_{n}, B \in \mathcal{M}_{n, m}, Q \in \mathcal{M}_{n, q}$ and $C \in \mathcal{M}_{p, n}$.

With the notation in the introduction, our problem can be stated as follows: Find, for a generic $Q$, the maximal dimension of $\operatorname{Im} C$ so that $\mathfrak{Y}(A, B ; \operatorname{Ker} C) \supset \operatorname{Im} Q$.

Taking into account that

$$
\operatorname{Im} Q \subset \mathfrak{Y}(A, B ; \operatorname{Ker} C) \subset \operatorname{Ker} C \text {, }
$$

finding the maximal dimension for $\operatorname{Im} C$ is equivalent to obtain the minimal dimension for $\operatorname{Ker} C$ so that $\operatorname{Ker} C$ is an $(A, B)$-invariant subspace containing $\operatorname{Im} Q$. In turns, this is equivalent to finding the minimal dimension of $\operatorname{Ker} C$ such that $(\operatorname{Ker} C)^{\perp}$ is a $\left(B^{*}, A^{*}\right)$-invariant subspace of maximal dimension contained in $(\operatorname{Im} Q)^{\perp}$. We recall that a subspace $S$ is said to be $\left(B^{*}, A^{*}\right)$-conditioned invariant (or $\left(\begin{array}{l}A^{*} \\ B^{*}\end{array}\right)$-invariant as in [3]) if $S^{\perp}$ is $(A, B)$-invariant.

We are going to describe a method of obtaining this dimension. We will divide our procedure in several steps:

(i) Since the matrix $Q$ is supposed to be generic, that is to say, belonging to an open and dense subset of $\mathcal{M}_{n, q}$ we can assume that $Q$ is a full rank matrix. Then, through 
a convenient change of bases, $Q$ takes the form

$$
Q=\left(\begin{array}{c}
-P^{*} \\
I_{q}
\end{array}\right)
$$

where $P \in \mathcal{M}_{q, n-q}$. Matrices $A, B$ and $C$ modify accordingly to this change of bases, but we will keep on with the same notation for these new matrices.

Then,

$$
(\operatorname{Im} Q)^{\perp}=\left[\begin{array}{l}
I_{n-q} \\
P
\end{array}\right]
$$

Call this subspace $F$.

(ii) Let $\left(\begin{array}{l}N \\ E\end{array}\right)$ be the Brunovsky (dual) form of the pair $\left(\begin{array}{l}A^{*} \\ B^{*}\end{array}\right)$ (see for example [3] (6.6.3)), $\left(\begin{array}{c}M \\ F\end{array}\right)$ a $B$-matrix and $\operatorname{Inv}\left(\left(B^{*}, A^{*}\right) ;(M, F)\right)$ the set of $\left(B^{*} A^{*}\right)$-conditioned invariant subspaces $W$ such that the restriction of $\left(\begin{array}{c}A^{*} \\ B^{*}\end{array}\right)$ to $W$ has a fixed Brunovsky form $\left(\begin{array}{l}M \\ F\end{array}\right)$ (see [2] for the definition of this restriction).

We denote by $\underline{k}=\left(k_{1} \geq k_{2} \geq \ldots \geq k_{r}\right)$ the observability indices of $\left(\begin{array}{l}N \\ E\end{array}\right)$ and for every eigenvalue $\lambda$ of $N_{\infty}, \eta_{1}(\lambda) \geq \eta_{2}(\lambda) \geq \ldots$ is the corresponding Segre characteristic. Analogously, $\underline{h}=\left(h_{1} \geq h_{2} \geq \ldots \geq h_{s}\right)$ and $\varepsilon_{1}(\lambda) \geq \varepsilon_{2}(\lambda) \geq \ldots$ are respectively the observability indices of $\left(\begin{array}{c}M \\ F\end{array}\right)$ and the Segre characteristic of $\lambda$ as eigenvalue of $M_{\infty}$. We assume that $\left(\begin{array}{c}M \\ F\end{array}\right)$ is compatible with $\left(\begin{array}{l}N \\ E\end{array}\right)$, that is to say that Inv $\left(\left(B^{*}, A^{*}\right) ;(M, F)\right)$ is not empty. We recall that a $B$-matrix $\left(\begin{array}{c}M \\ F\end{array}\right)$ is compatible with $\left(\begin{array}{l}N \\ E\end{array}\right)$ if and only if the following conditions hold (see for example [1]):

(a) $s<r$, and $h_{i} \leq k_{i}$ for $i=0,1,2, \ldots$

(b) the eigenvalues of $M_{\infty}$ are also eigenvalues of $N_{\infty}$, and for each one the corresponging Segre characteristics $\left(\eta_{1}(\lambda), \eta_{2}(\lambda), \ldots\right)$ and $\left(\varepsilon_{1}(\lambda), \varepsilon_{2}(\lambda), \ldots\right)$ verify: $\eta_{i}(\lambda) \leq \varepsilon_{i}(\lambda)$, for $i=1,2, \ldots$

In [2] (4.6) (see also [4] (2.9)) the following formula is obtained:

$$
\begin{aligned}
\operatorname{dim}\left(\left(B^{*}, A^{*}\right) ;(M, F)\right)= & \sum_{i=1}^{h} \sum_{j=1}^{k} m_{i}\left(r_{i+j-1}-s_{i+j-1}\right)+ \\
& +\sum_{\lambda} \sum_{i} e_{i}(\lambda)\left(c_{i}(\lambda)-e_{i}(\lambda)\right)+\left(k_{\infty}-h_{\infty}\right) s
\end{aligned}
$$

where, $m_{i}=s_{i}-s_{i+1}, \underline{r}=\left(r_{1} \geq \ldots \geq r_{k}\right)$ is the conjugate partition of $\underline{k}=\left(k_{1} \geq\right.$ $\left.\ldots \geq k_{r}\right), \underline{s}=\left(s \geq \ldots \geq s_{h}\right)$ is the conjugate partition of $\underline{h}=\left(h_{1} \geq \ldots \geq h_{s}\right)$, $\lambda$ runs over the eigenvalues of $M_{\infty}, c_{1}(\lambda) \geq c_{2}(\lambda) \geq \ldots, e_{1}(\lambda) \geq e_{2}(\lambda) \geq \ldots$ are the conjugate partitions of the Segre characteristics $\eta_{1}(\lambda) \geq \eta_{2}(\lambda) \geq \ldots, \varepsilon_{1}(\lambda) \geq$ $\varepsilon_{2}(\lambda) \geq \ldots$ of $\lambda$ in $N_{\infty}$ and $M_{\infty}$, respectively, and $k_{\infty}, h_{\infty}$ are the size of $N_{\infty}$ and $M_{\infty}$, respectively. Set

$$
\operatorname{dim}\left(\left(B^{*}, A^{*}\right) ;(M, F)\right)=\delta
$$


(iii) Now, we have to find, for the $Q$ given in (i), the maximal dimension $d$ so that there exists a $d$-dimensional $\left(B^{*} A^{*}\right)$-conditioned invariant subspace contained in $F$. This leads to consider the intersection

$$
\operatorname{Gr}_{d}(F) \cap \operatorname{Inv}\left(\left(B^{*}, A^{*}\right) ;(M, F)\right)
$$

for all possible $\left(\begin{array}{l}M \\ F\end{array}\right)$ compatibles with $\left(\begin{array}{l}N \\ E\end{array}\right)$ such that $M \in \mathcal{M}_{d}$.

We know by Thom Transversality theorem that if

$$
\operatorname{dim} \operatorname{Gr}_{d}(F)+\delta<\operatorname{dim} \operatorname{Gr}_{d}\left(\mathbb{C}^{n}\right)
$$

the intersection $\operatorname{Gr}_{d}(F) \cap \operatorname{Inv}\left(\left(B^{*}, A^{*}\right) ;(M, F)\right)$ will be empty for $Q$ generic. So, we have to look for the greatest $d$ such that

$$
d(n-q-d)+\delta \geq d(n-d)
$$

for some compatible $\left(\begin{array}{c}M \\ F\end{array}\right)$, or equivalently

$$
\delta \geq d q
$$

Then, we have the following result

Theorem 1 With the above notation, the maximal dimension of the $\left(B^{*}, A^{*}\right)$-conditioned invariant subspaces contained in $(\operatorname{Im} Q)^{\perp}$ is the greatest $d$ such that $\delta \geq d q$ and the set of such subspaces is a manifold of dimension $\delta-d q$. Besides of this, the Brunovsky reduced form of the restriction of $\left(B^{*} A^{*}\right)$ to these subspaces is of the same type of $\left(\begin{array}{c}M \\ F\end{array}\right)$ (that is to say, they have the same observability indices and for each eigenvalue of the Jordan part the Segre characteristic is the same).

Example 2 We are going to apply this theorem to the following situation: $\underline{k}=(5,2)$, so that $\underline{r}=\left(\begin{array}{llll}2 & 2 & 1 & 1\end{array}\right), c=3$ and $q=2$. We begin for the possible maximal dimension: $d=8$. In this case the only possible cases are:

$$
\left(\begin{array}{lllll}
2 & 2 & 1 & 1 & 1
\end{array}\right), 1 ; \quad(2 \quad 2 \quad 11), 2 ; \quad \text { and } \quad(22), 3,
$$

for $\underline{s}$ and $e$, respectively, and it easily checked that in any case is $\delta<15$. Continuing this way we see that for $d=4$ and $s=(2,2), e=0$ one has that $\delta=8$. Hence, in this example the maximal solution of the DDP is obtained for $d=4$. Notice that if $s=(2,1), e=1$, then $\delta=10$. That is to say, the maximal dimension is unique but not the Brunovsky form of the restriction to the corresponding invariant subspaces.

(2.2) It is also possible to give a method for obtaining a parametrization of the manifold

$$
\operatorname{Gr}_{d}(F) \cap \operatorname{Inv}\left(\left(B^{*}, A^{*}\right) ;(M, F)\right) .
$$

However, only when the pair $(A, B)$ is controllable, or equivalently $\left(\begin{array}{l}A^{*} \\ B^{*}\end{array}\right)$ is observable, the procedure can be given in an explicit way. So we limit ourselves to consider this case. We will use the explicit description of a coordinate system given in [4] (3.3). There it is 
shown that, with the notation in (2.1), for any set of positive integers pairwise different $\left\{n_{i j}, 1 \leq i \leq h, i \leq j \leq m_{h-i+1}\right\}$ such that for $i=1,2, \ldots, h$

$$
1 \leq n_{i 1}<n_{i 2}<\ldots<n_{i m_{h-i+1}} \leq r_{h-i+1}
$$

if $Y=\left(Y_{i j}\right), 1 \leq i \leq k, 1 \leq j \leq h$ is a matrix verifying the conditions below, then the free parameters in $Y$ define a coordinate system in Inv $\left(\left(B^{*}, A^{*}\right) ;(M, F)\right)$. The conditions are the following:

1. $Y_{i j} \in \mathcal{M}_{r_{i}, s_{j}}$.

2. $Y_{i j}=0$ if $i<j$.

3. For $i=1,2, \ldots, h, Y_{i i}$ is partitioned into blocks $Y_{i i}=\left(L_{i \beta}^{1}\right), 1 \leq \beta \leq h-i+1$ in such a way that for $\beta=1,2, \ldots, h-i+1, L_{i \beta}^{1} \in \mathcal{M}_{r_{i}, m_{h-\beta+1}}$ is a matrix whose last $r_{i}-r_{h-\beta+1}$ rows are zero, the rows $n_{i j}, 1 \leq i \leq \beta-1,1 \leq j \leq m_{h-i+1}$ are also zero, and the rows $n_{\beta 1}, n_{\beta 2}, \ldots, n_{\beta m_{h-\beta+1}}$ are unit vectors $e_{1}^{\beta}, e_{2}^{\beta}, \ldots, e_{m_{h-\beta+1}}^{\beta}$ :

$$
e_{j}^{\beta}=(0, \ldots, 0, \stackrel{(j}{1}, 0, \ldots, 0) \in(\mathbb{F})^{m_{h-\beta+1}} .
$$

4. For $i>j, Y_{i j}$ is partitioned into blocks $Y_{i j}=\left(L_{i \beta}^{i-j+1}\right), 1 \leq \beta \leq h-j+1$ in such a way that $L_{i \beta}^{i-j+1} \in \mathcal{M}_{r_{i}, m_{h-\beta+1}}$ is a matrix whose last $r_{i}-r_{h-\beta+i-j+1}$ rows are zero and for $\beta \geq i-j+1$, the rows $n_{p q}, 1 \leq p \leq \beta-i+j, 1 \leq q \leq m_{h-p+1}$ are also zero. (Notice that $Y_{i j}$ follows from $Y_{i-j+1,1}$ ).

If $Y$ is such a matrix, the coordinate chart is simply given by the mapping

$$
\psi: \mathbb{C}^{\delta} \longrightarrow \operatorname{Inv}\left(\left(B^{*}, A^{*}\right) ;(M, F)\right)
$$

defined by $\psi($ free parameters in $Y)=[Y]$.

Then, taking into account that $F=\left[\begin{array}{l}I_{n-q} \\ P\end{array}\right]$, it turns out that a subspace $S$ belongs to $\operatorname{Gr}_{d}(F) \cap \operatorname{Inv}\left(\left(B^{*}, A^{*}\right) ;(M, F)\right)$, where we recall that $d$ and $(M, F)$ have been obtained in (iii), if and only if there exists $X \in \mathcal{M}_{n-q, d}^{*}$ such that

$$
Y=\left(\begin{array}{l}
I_{n-q} \\
P
\end{array}\right) X .
$$

So, if we write $Y=\left(\begin{array}{l}Y_{1} \\ Y_{2}\end{array}\right)$ where $Y_{1} \in \mathcal{M}_{n-q, d}$, then $X=Y_{1}$ and

$$
Y_{2}-P Y=0
$$

This is a linear system of $q \cdot d$ equations with $\delta$ unknowns. Hence, for $Q$ generic the solutions depend on $(\delta-q) \cdot \delta$ free parameters, which is just the dimension of the manifold $\operatorname{Gr}_{d}(F) \cap \operatorname{Inv}\left(\left(B^{*}, A^{*}\right) ;(M, F)\right)$, so we have proved the following result:

Proposition 3 With the above notation, the mapping which assigns to each set of $(\delta-q) \cdot d$ free parameters in the linear system $Y_{2}-P Y_{1}=0$ the subspace $[Y]$, where $Y=\left(\begin{array}{l}Y_{1} \\ Y_{2}\end{array}\right)$ is the corresponding solution of this system, defines a coordinate chart in $G r_{d}(F) \cap \operatorname{Inv}\left(\left(B^{*}, A^{*}\right) ;(M, F)\right)$. 


\section{References}

[1] I. Baragaña, I. Zaballa, Block Similarity Invariants of Restrictions to $(A, B)$ Invariants Subspaces. Linear Algebra and Appl., (220): 31-62, 1995.

[2] J. Ferrer, F. Puerta, X. Puerta, Stratification of the set of general $(A, B)$-invariant subspaces. Preprint (1999).

[3] I. Gohberg, P. Lancaster, L. Rodman, Invariant Subspaces of Matrices with Applications. Wiley, New York, 1986.

[4] F. Puerta, X. Puerta, I. Zaballa, A coordinate atlas of the manifold of observable conditioned invariant subspaces. Preprint (2000).

[5] W.M. Wonham, Linear Multivariable Control: A Geometrical Approach. SpringerVerlag, New York, 1984. 